\title{
Molecular characterization of a novel ssRNA ourmia-like virus from the rice blast fungus
}

\section{Magnaporthe oryzae}

Adriana Illana ${ }^{1,2}$, Marco Marconi ${ }^{1,2}$, Julio Rodríguez-Romero ${ }^{1,2}$, Ping $\mathrm{Xu}^{3}$, Tamas Dalmay ${ }^{3}$, Mark Wilkinson $^{1,2}$, Maria Ángeles Ayllón ${ }^{1,2}$, and Ane Sesma ${ }^{1,2}$

${ }^{1}$ Centro de Biotecnología y Genómica de Plantas. Universidad Politécnica de Madrid (UPM) - Instituto Nacional de Investigación y Tecnología Agraria y Alimentaria (INIA Campus Montegancedo UPM) 28223-Pozuelo de Alarcón (Madrid), Spain; ${ }^{2}$ Departamento de Biotecnología-Biología Vegetal, Escuela Técnica Superior de Ingeniería Agronómica, Alimentaria y de Biosistemas, UPM, 28040Madrid, Spain. ${ }^{3}$ School of Biological Sciences, University of East Anglia, Norwich Research Park, Norwich, NR4 7TJ (UK).

*Correspondence: ane.sesma@upm.es; phone:+34 913364593.

\begin{abstract}
In this study we characterize a novel positive and single stranded RNA (ssRNA) mycovirus isolated from the rice field isolate of Magnaporthe oryzae Guy11. The ssRNA contains a single open reading frame (ORF) of 2,373 nucleotides in length and encodes an RNA-dependent RNA polymerase (RdRp) closely related to ourmiaviruses (plant viruses) and ourmia-like mycoviruses. Accordingly, we name this virus Magnaporthe oryzae ourmia-like virus 1 (MOLV1). Although phylogenetic analysis suggests that MOLV1 is closely related to ourmia and ourmia-like viruses, it has some features never reported before within the Ourmiavirus genus. 3' RLM-RACE (RNA ligase-mediated rapid amplification of cDNA ends) and extension poly(A) tests (ePAT) suggest that the MOLV1 genome contains a poly(A) tail whereas the three cystein and the three guanine residues present in 5' and 3' untranslated regions (UTRs) of ourmia viruses are not observed in the MOLV1 sequence. The discovery of this novel viral genome supports the hypothesis that plant pathogenic fungi may have acquired this type of viruses from their host plants.
\end{abstract}

\section{INTRODUCTION}

Mycoviruses are widespread in all taxonomic groups of fungi. Different outcomes result from the interaction between mycoviruses and their respective fungal hosts. Usually, a mycovirus infection remains latent and shows no symptoms in the fungal host [9]. Other mycoviruses are able to cause severe lesions and impair the vegetative growth of their fungal host, causing economic losses as in the case of the cultivated mushroom Agaricus bisporus [9, 10]. Presence of a mycovirus can also be beneficial for all the partners in the tripartite interaction of virus, fungus and plant host. This is the case 
described for a tropical panic grass and its fungal endophyte when infected by a virus, which increases its survival under stress temperature conditions [21]. The mycovirus can additionaly cause fungal hypovirulence in the host plant making it a good option for biological control, as is the case for the well-known Criphonectria hypovirus 1 (CHV1) and chestnut blight control in Europe [24, 37].

Mycoviruses have a diverse genome organization. Most viruses have double stranded RNA (dsRNA) or linear positive (+) ssRNA genomes [9]. Unclassified linear negative ssRNAs genomes and circular ssDNA viruses have also been isolated [10, 19, 22, 23]. Linear (+) ssRNA mycoviruses are classified into 5 families: Alphaflexiviridiae, Gammaflexiviridae, Hypoviridae, Narnaviridae and Barnaviridae [2, 10]. The Narnaviridae family includes the Mitovirus and Narnavirus genera.

The (+) ssRNA genomes from Narnaviridae family are the simplest mycoviruses. Members of this family have a 2-3 Kb genome size, which usually encodes an RNA-dependent RNA polymerase (RdRp) needed for their own replication. Narnaviruses lack the coat protein (CP) that forms the capside structure or the movement protein (MP) [11]. Phylogenetically, the narnaviruses are the closest relatives of the Ourmiavirus plant genus [11, 27]. Plant ourmiaviruses contain a tripartite ssRNA genome that codes for three proteins, the RdRp, the CP and the MP [27]. Phylogenetic analyses support the idea that the Ourmiavirus genus represents a link between mycoviruses and plant viruses [6, 11].

The ascomycetous fungus Magnaporthe oryzae causes the damaging rice blast disease and results in approximately 30\% yield loss in rice fields every year [29]. M. oryzae was the first plant pathogenic fungus where the presence of a polyhedral virus was reported [14, 38]. Several viruses from different families have been found in this fungal species. Magnaporthe oryzae virus 1, 2 and 3 (MoV1, MoV2 and MoV3) are dsRNA viruses belonging to Totiviridae family [20, 31, 39]. Magnaporthe oryzae chrysovirus 1A and B (MoCV1-A and MoCV1-B) also are dsRNA viruses that belong to the Chrysoviridae family. They can impair vegetative and invasive growth during $M$. oryzae host colonisation [32-34]. Recently, a (+) ssRNA virus has been described for the first time in this fungal species [1], Magnaporthe oryzae virus A (MoVA). The genome of MoVA is 3,246 nt in length and contains two in-frame ORFs, one of which encodes a protein with similarity to an RdRp observed in plant viruses of the Tombusviridae family.

In this work we describe a second and novel type of ssRNA virus found in the $M$. oryzae rice isolate Guy11, Magnaporthe oryzae ourmia-like virus 1 (MOLV1), a polyadenylated (+) ssRNA virus phylogenetically related to plant viruses from the Ourmiavirus genus.

\section{RESULTS}

\section{Discovery of a new mycovirus in Magnaporthe oryzae}


During the analysis of sequences derived from small RNA libraries isolated from the rice field isolate Guy11 [17], we detected sequences that were not present in the published $M$. oryzae genome of the laboratory strain 70-15 [5], a near-isogenic strain of Guy11. We additionally confirmed this by downloading EST sequences from M. oryzae Guy11 and aligning them to the reference genome. EST sequences that failed to align were collected and assembled using the Cap3 DNA sequence assembly program [13]. Using these unknown ESTs, we predicted putative ORFs using the getorf tool from the EMBOSS suite [28], which were aligned against the NCBI non-redundant sequence collection [25] by blastp [4]. This allowed us to identify transcripts showing similarities with ourmia-like mycoviruses, and plant viruses of the Ourmiavirus genus. Consequently, we named this viral genome Magnaporthe oryzae ourmia-like virus 1 (MOLV1).

Presence of MOLV1 RNA virus in M.oryzae Guy11 strain was confirmed by Northern blotting using total RNA extracts (Fig. 1A). Almost all of the retrieved MOLV1 sequence was used as a DNA probe, and actin DNA was used as the control. A band of aproximately $2.3 \mathrm{~Kb}$ in the M. oryzae Guy11 strain was observed, in addition to other smaller bands. These RNA molecules were not detected in the M. oryzae laboratory strain $70-15$. This is consistent with the absence of these viral sequences from EST collections other than those of the fungal isolate Guy11.

\section{3' RLM-RACE and ePAT experiments suggest that MOLV1 is a polyadenylated $(+)$ ssRNA virus}

To identify 5' and 3' untranslated regions (UTRs) of MOLV1 we carried out an RNA ligase-mediated rapid amplification of cDNA ends (RLM-RACE) (Fig.1B). The 3' terminus is usually polyadenylated before conducting a 3' RLM-RACE in non-polyadenylated viruses. In our case we obtained a clear band without adding any adenosine to the RNA sample. We purified and sequenced the largest bands obtained from both 5' and 3' RLM-RACE experiments (asterisks, Fig. 1B). The 5' and 3' UTRs of MOLV1 are $116 \mathrm{nt}$ and $430 \mathrm{nt}$ in length, respectively. To confirm the presence of a poly(A) tail in the MOLV1 genome an extension poly(A) test (ePAT) experiment was carried out (Fig.1C). This method allows measurement of the poly(A) tail length of polyadenylated RNA molecules [15]. The difference in band sizes between MOLV1 and the negative control TVN-PAT indicated that the poly(A) tail of MOLV1 is between 25-30 nt in length.

These experiments suggested that the MOLV1 genome is a positive and polyadenylated ssRNA molecule of 2,364 nt, excluding the poly(A) tail. We also performed the ePAT experiment to determine if the BOLV genome contained a poly(A) tail and found that it did not. With the exception of $S$. sclerotiorum mitovirus 1/KL-1 (SsMV-1/KL1) and 2a (CcMV-2a), no other members of the family Narnaviridae are polyadenylated at their 3' end [11, 36]. To our knowledge, this is the first described ourmia-like mycovirus that has a poly(A) tail. However, these results should be treated cautiously since 
it is possible to find amplification products with oligodT primers in non-polyadenylated RNA viruses due to the presence of small fractions of gRNA with poly(A) tails [18, 26].

The MOLV1 genome has a unique open reading frame (ORF) from position 117 to 1931 (Fig.1D). The translation of this $818 \mathrm{nt}$ ORF generates a polypeptide of 605 aa with a theoretical molecular weight of $67.12 \mathrm{kDa}$. This protein has higher identity with the RdRp of Rhizoctonia solani ourmia-like virus 1 (RsOLV1; $40 \%$ ), although it is also similar to other ourmia-like mycoviruses and plant ourmiaviruses (Fig.2). The MOLV1 viral protein contains the conserved domains of the RdRp present in (+) ssRNA viruses [16], including the highly conserved core domain GDD in motif VI (Fig.2A). These findings suggest that the ORF of MOLV1 encodes a putative RdRp. The amino acid alignment of MOLV1 with the BOLV sequence showed no conservation of the three cystein residues at the 5' UTR and the three guanine residues at the 3' UTR. The predicted secondary structure indicated the presence of a stable terminal stem-loop structure only for the first $40 \mathrm{nt}$ of MOLV1 ( $\Delta \mathrm{G}$ value of $-10.00 \mathrm{kcal} / \mathrm{mol}$ ) (Fig.S1A). The last $34 \mathrm{nt}$ of the 3' UTR could be folded into a less stable stem-loop structure with $\Delta \mathrm{G}$ value of $-6.30 \mathrm{kcal} / \mathrm{mol}$. These secondary structures at both UTRs could play a role in the replication of MOLV1 by providing a recognition site for RdRp and/or protecting the genome from degradation as has been proposed for BOLV and mitoviruses [11, 12, 36].

\section{MOLV1 is closely related to plant ourmiaviruses and ourmia-like mycoviruses}

Full-length amino acid sequences of published RdRps (29 proteins) were selected to construct a phylogenetic tree to infer the evolutionary history of MOLV1 (Fig.2B). The RdRp of the tobamovirus tobacco mosaic virus (TMV) was used as an outgroup in the tree. The maximum likelihood (ML) phylogenetic tree was inferred from the amino acid sequences of viral RdRps after removing positions with less than $50 \%$ site coverage. The phylogenetic analyses clearly showed two clades. The first clade grouped all mitoviruses. The second clade grouped the two Saccharomyces narnaviruses and Phytophthora infestans RNA virus (82\% bootstrap support), and all the ourmia-like mycoviruses and the plant ourmiaviruses (97\% bootstrap support). This ML analysis indicates that fungal ourmia-like viruses are more closely related to plant viruses of the Ourmiavirus genus than mycoviruses of the Narnaviridae family. As previously shown, this phylogenetic tree places ourmia-like viruses as more closely related to members of the genus Narnavirus than to members of the genus Mitovirus [3, 6, 27].

Different theories have been suggested for mycoviruses evolution [8]. The plant ourmiavirus genomes have three RNA molecules which encode three proteins, the RdRp, the CP and the MP [27]. The identification of ourmia-like mycoviruses such as MOLV1, which contain a single ORF and being closely related to plant ourmia viruses suggest that they might have been originated by gene loss events during their adaptation to the fungal host as has been suggested for other ourmia-like mycoviruses [6, 
22, 23]. However, we cannot discard the possibility of an ourmia-like mycovirus being the ancestor of plant ourmiaviruses as it has been proposed [27].

In summary, our results suggest that MOLV1 is a novel (+) ssRNA virus isolated from the rice blast fungus closely related to ourmia-like mycoviruses and plant ourmia viruses that exhibits new features such as a poly(A) tail and showed no conservation of the three cystein residues at the 5' UTR and the three guanine residues at the 3' UTR.

\section{Acknowledgments}

A.S. is funded by the Spanish Research council (grant ref. BIO2014-53211-R), the EC (REA grant agreement n. 304039) and the Community of Madrid (grant ref. S2013/ABI-2734).

\section{Compliance with ethical standards and conflict of interest}

This paper is in compliance with ethical standards for research. The authors declare no conflicts of interest.

\section{References}

1. Ai Y-P, Zhong J, Chen C-Y, Zhu H-J, Gao B-D (2016) A novel single-stranded RNA virus isolated from the rice-pathogenic fungus Magnaporthe oryzae with similarity to members of the family Tombusviridae. Archives of Virology 161:725-729

2. Andrew King EL, Michael J. Adams and Eric B. Carstens (2012) Virus Taxonomy, 1st Edition, Ninth Report of the International Committee on Taxonomy of Viruses. Elsevier:1344

3. Cai G, Krychiw JF, Myers K, Fry WE, Hillman BI (2013) A new virus from the plant pathogenic oomycete Phytophthora infestans with an $8 \mathrm{~kb}$ dsRNA genome: The sixth member of a proposed new virus genus. Virology 435:341-349

4. Camacho C, Coulouris G, Avagyan V, Ma N, Papadopoulos J, Bealer K, Madden TL (2009) BLAST+: architecture and applications. BMC Bioinformatics 10:421-421

5. Chao CCT, Ellingboe AH (1991) Selection for mating competence in Magnaporthe grisea pathogenic to rice. Canadian Journal of Botany/Revue Canadienne de Botanique 69:130-134

6. Donaire L, Rozas J, Ayllón MA (2016) Molecular characterization of Botrytis ourmia-like virus, a mycovirus close to the plant pathogenic genus Ourmiavirus. Virology 489:158-164

7. Edgar RC (2004) MUSCLE: multiple sequence alignment with high accuracy and high throughput. Nucleic Acids Research 32:1792-1797

8. Ghabrial SA (1998) Origin, adaptation and evolutionary pathways of fungal viruses. Virus Genes 16 (1):119-131

9. Ghabrial SA, Suzuki N (2009) Viruses of Plant Pathogenic Fungi. Annual Review of Phytopathology 47:353-384

10. Ghabrial SA, Castón JR, Jiang D, Nibert ML, Suzuki N (2015) 50-plus years of fungal viruses. Virology 479-480:356-368

11. Hillman BI, Cai G (2013) Chapter Six - The Family Narnaviridae: Simplest of RNA Viruses. In: Said AG (ed) Advances in Virus Research. Academic Press, pp 149-176 
12. Hong Y, Dover SL, Cole TE, Brasier CM, Buck KW (1999) Multiple Mitochondrial Viruses in an Isolate of the Dutch Elm Disease Fungus Ophiostoma novo-ulmi. Virology 258:118-127

13. Huang X, Madan A (1999) CAP3: A DNA Sequence Assembly Program. Genome Research 9:868877

14. Hunst PL. LHF, Rossi AE (1986) Variation in a double-stranded RNA from isolates of Pyricularia oryzae. Phytopathology 76:674-678

15. Jänicke A, Vancuylenberg J, Boag PR, Traven A, Beilharz TH (2012) ePAT: A simple method to tag adenylated RNA to measure poly(A)-tail length and other 3 ' RACE applications. RNA 18:12891295

16. Koonin EV (1991) The phylogeny of RNA-dependent RNA polymerases of positive-strand RNA viruses. Journal of General Virology 72:2197-2206

17. Leung H, Borromeo E, Bernardo M, Notteghem JL (1988) Genetic analysis of virulence in the rice blast fungus Magnaporthe grisea. Phytopathology 78:1227-1233

18. Li W, Zhang Y, Zhang C, Pei X, Wang Z, Jia S (2014) Presence of poly(A) and poly(A)-rich tails in a positive-strand RNA virus known to lack 3' poly(A) tails. Virology 454-455:1-10

19. Liu L, Xie J, Cheng J, Fu Y, Li G, Yi X, Jiang D (2014) Fungal negative-stranded RNA virus that is related to bornaviruses and nyaviruses. Proceedings of the National Academy of Sciences of the United States of America 111:12205-12210

20. Maejima K, Himeno M, Komatsu K, Kakizawa S, Yamaji Y, Hamamoto H, Namba S (2008) Complete nucleotide sequence of a new double-stranded RNA virus from the rice blast fungus, Magnaporthe oryzae. Archives of Virology 153:389-391

21. Márquez LM, Redman RS, Rodriguez RJ, Roossinck MJ (2007) A Virus in a Fungus in a Plant: Three-Way Symbiosis Required for Thermal Tolerance. Science 315:513-515

22. Marzano S-YL, Domier LL (2016) Novel mycoviruses discovered from metatranscriptomics survey of soybean phyllosphere phytobiomes. Virus Research 213:332-342

23. Marzano S-YL, Nelson BD, Ajayi-Oyetunde O, Bradley CA, Hughes TJ, Hartman GL, Eastburn DM, Domier LL (2016) Identification of diverse mycoviruses through metatranscriptomics characterization of the viromes of five major fungal plant pathogens. Journal of Virology

24. Nuss DL (2005) Hypovirulence: Mycoviruses at the fungal-plant interface. Nat Rev Micro 3:632642

25. Pruitt KD, Tatusova T, Maglott DR (2005) NCBI Reference Sequence (RefSeq): a curated nonredundant sequence database of genomes, transcripts and proteins. Nucleic Acids Research 33:D501-D504

26. Raju R, Hajjou M, Hill KR, Botta V, Botta S (1999) In vivo addition of poly(A) tail and AU-rich sequences to the 3' terminus of the Sindbis virus RNA genome: a novel 3'-end repair pathway. Journal of Virology 73:2410-2419

27. Rastgou M, Habibi MK, Izadpanah K, Masenga V, Milne RG, Wolf YI, Koonin EV, Turina M (2009) Molecular characterization of the plant virus genus Ourmiavirus and evidence of interkingdom reassortment of viral genome segments as its possible route of origin. Journal of General Virology 90:2525-2535

28. Rice P, Longden I, Bleasby A (2000) EMBOSS: The European Molecular Biology Open Software Suite. Trends in Genetics 16:276-277

29. Skamnioti P, Gurr SJ (2009) Against the grain: safeguarding rice from rice blast disease. Trends in Biotechnology 27:141-150

30. Tamura K, Stecher G, Peterson D, Filipski A, Kumar S (2013) MEGA6: Molecular Evolutionary Genetics Analysis Version 6.0. Molecular Biology and Evolution 30:2725-2729

31. Tang L, Hu Y, Liu L, Wu S, Xie J, Cheng J, Fu Y, Zhang G, Ma J, Wang Y, Zhang L (2015) Genomic organization of a novel victorivirus from the rice blast fungus Magnaporthe oryzae. Arch Virol

32. Urayama S, Kato S, Suzuki Y, Aoki N, Le MT, Arie T, Teraoka T, Fukuhara T, Moriyama H (2010) Mycoviruses related to chrysovirus affect vegetative growth in the rice blast fungus Magnaporthe oryzae. Journal of General Virology 91:3085-3094

33. Urayama S, Ohta T, Onozuka N, Sakoda H, Fukuhara T, Arie T, Teraoka T, Moriyama H (2012) Characterization of Magnaporthe oryzae Chrysovirus 1 Structural Proteins and Their Expression in Saccharomyces cerevisiae. Journal of Virology 86:8287-8295 
34. Urayama S, Sakoda H, Takai R, Katoh Y, Minh Le T, Fukuhara T, Arie T, Teraoka T, Moriyama H (2014) A dsRNA mycovirus, Magnaporthe oryzae chrysovirus 1-B, suppresses vegetative growth and development of the rice blast fungus. Virology 448:265-273

35. Whelan S, Goldman N (2001) A General Empirical Model of Protein Evolution Derived from Multiple Protein Families Using a Maximum-Likelihood Approach. Molecular Biology and Evolution 18:691-699

36. Xie J, Ghabrial SA (2012) Molecular characterizations of two mitoviruses co-infecting a hyovirulent isolate of the plant pathogenic fungus Sclerotinia sclerotiorum. Virology 428:77-85

37. Xie J, Jiang D (2014) New Insights into Mycoviruses and Exploration for the Biological Control of Crop Fungal Diseases. Annual Review of Phytopathology 52:45-68

38. Yamashita S, Doi Y, Yora K (1971) A Polyhedral Virus Found in Rice Blast Fungus, Pyricularia oryzae Cavara. Japanese Journal of Phytopathology 37:356-359

39. Yokoi T, Yamashita S, Hibi T (2007) The nucleotide sequence and genome organization of Magnaporthe oryzae virus 1. Archives of Virology 152:2265-2269 


\section{Figure 1}

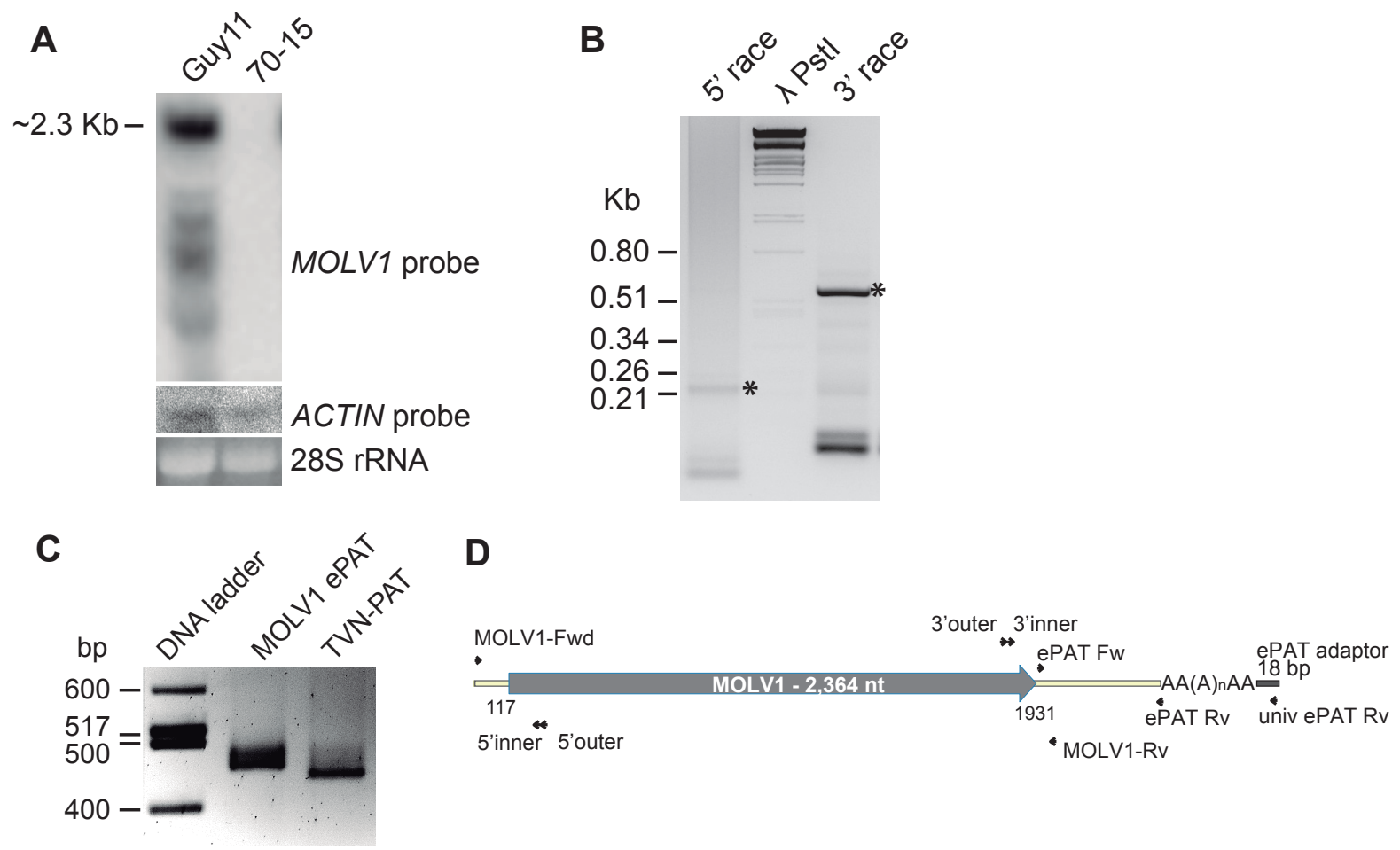

Fig.1. Characterisation of a novel RNA virus in the rice blast fungus. (A) Northern blots using $30 \mu \mathrm{g}$ of total RNA extracted from M. oryzae Guy11 grown on complete media. MOLV1 and actin probes were PCR products amplified with MOLV1_fw (5'GCTTTCGCTTCTTTGGTCGT3') / MOLV1_rv (5'GCTCCCTAACGTGGCGATAA3'), and Actin_fw (5'CTTCTCTCGGTTGGACTTGG3') / Actinrv (5'TTCTACAACGAGCTGCGTGT3') primers, respectively. The actin probe was used as hybridization control. Ethidium bromide gel staining of $28 \mathrm{~S}$ ribosomal RNA is shown as a loading control. (B) 5' and 3' RLM-RACE reactions were carried out using 5'Outer (5'ACTCTCAGATCAACCCTGAAGGT3') / 5'Inner (5'TCACTAAGCAGTCCAGAGAACGA3') and 3'Outer (5'AGGCGTATCGTCGAAAAGCCAA3') / 3'Inner (5'TTAAAGTTGTCGGTGAACGCGA3') primers, respectively. PCR products were loaded into 2\% agarose gel. Bands selected for cloning and further sequencing are indicated with asterisks. (C) ePAT experiment using total RNA from $M$. oryzae Guy11. PCR amplification of cDNA derived from $1 \mu \mathrm{g}$ of RNA sample using gene-specific primers for the virus (ePAT Fw 5'-GCCGGTCTGCTGTTGGGAT-3' and reverse 5'-TTTTTTTTTTTTCTTAAACCTACATGCTGGTC-3'). This ePAT reaction generated a fragment of aprox. $468 \mathrm{bp}$, which reflects the size of the 3'UTR plus the length of the poly(A) tail. The TVN-PAT reaction was used with the same forward primer and the universal ePAT reverse primer (5'GCGAGCTCCGCGGCCGCG-3'), which generated a fragment of aprox. 438 bp consistent with amplification of the 3'UTR without the poly(A) tail. PCR products from ePAT and TVN-PAT reactions were visualised in 2\% agarose gels. (D) Schematic representation of MOLV1 RNA genome showing the ORF, UTRs and location of primers used for ePAT and Northern experiments. 


\section{Figure 2}

A

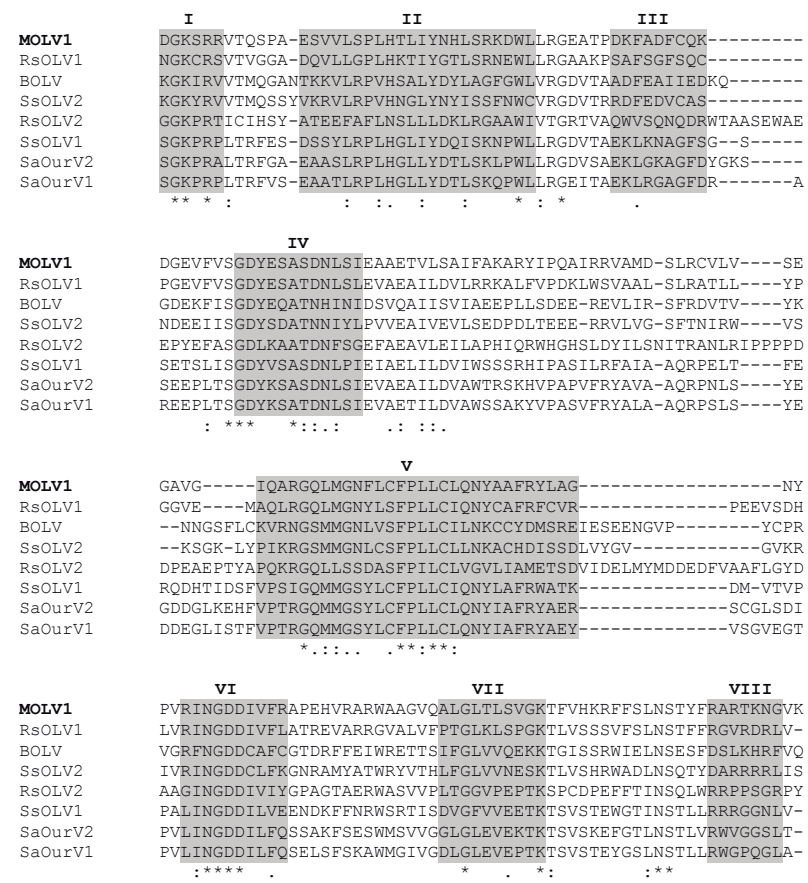

B

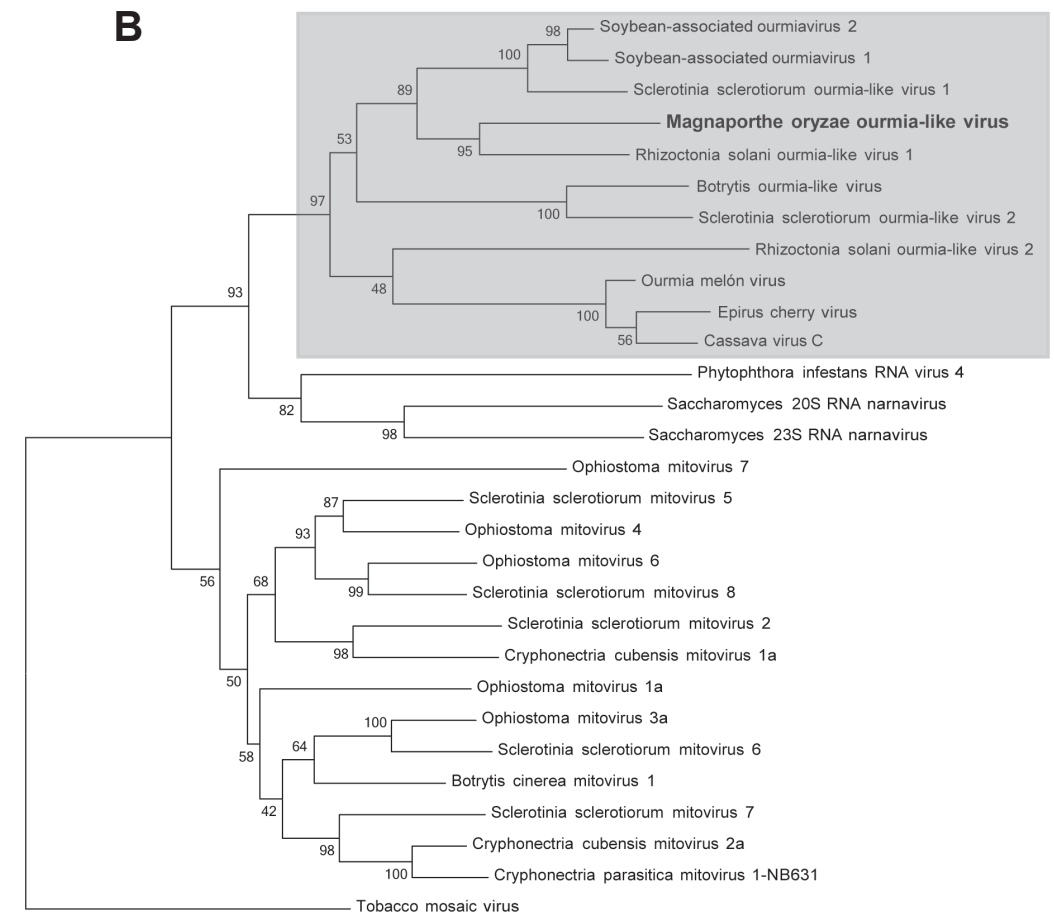

Fig.2. Sequence analysis of MOLV1. (A) Alignment showing RdRp conserved motifs I to VIII of MOLV1 (LT593139), RsOLV1 (ALD89131.1), BOLV (LN827955), SsOLV2 (ALD89139.1), RsOLV2 (ALD89132.1), SsOLV1 (ALD89138.1), SaOurV2 (KT598247.1) and SaOurV1 (KT598235.1). (B) Phylogenetic tree obtained by the Maximum Likelihood method with the RdRp sequences of 25 mycoviruses, including MOLV1, and 3 plant ourmiaviruses. The RdRp sequence of TMV (ABN79257.1) was included as an outgroup. The gray box shows ourmia-like fungal viruses. All bootstrap values (\%) are represented at each node of the tree (test with 1000 replicates). Branch lengths are proportional to the number of amino acid substitutions and are measured by the scale bar. Sequence accession numbers of viruses used are: Botrytis ourmia-like virus (LN827955), Soybean-associated ourmiavirus 1 (KT598235.1), Soybean-associated ourmiavirus 2 (KT598247.1), Sclerotinia sclerotiorum ourmia-like virus 1 (ALD89138.1), Sclerotinia sclerotiorum ourmia-like virus 2 (ALD89139.1), Rhizoctonia solani ourmia-like virus 1 (ALD89131.1) Rhizoctonia solani ourmia-like virus 2 (ALD89132.1), Sclerotinia sclerotiorum mitovirus 6 (AHX84133.1), Ophiostoma mitovirus 3a (NP_660176.1), Sclerotinia sclerotiorum mitovirus 7 (AHE13866.1), Botrytis cinerea mitovirus 1 (YP_002284334.1), Cryphonectria parasitica mitovirus 1-NB631 (NP_660174.1), Cryphonectria cubensis mitovirus 2a (AAR01973.1), Ophiostoma mitovirus 1a (CAJ32466.1), Sclerotinia sclerotiorum mitovirus 2 (AEX91879.1), Cryphonectria cubensis mitovirus 1a (AAR01970.1), Sclerotinia sclerotiorum mitovirus 8 (AHE13867.1), Ophiostoma mitovirus 6 (NP_660181.1), Sclerotinia sclerotiorum mitovirus 5 (AHX84130.1), Ophiostoma mitovirus 4 (NP_660179.1), Phytophthora infestans RNA virus 4 (AEM89293.1), Saccharomyces 20S RNA narnavirus (NP_660178.1), Saccharomyces 23S RNA narnavirus (NP_660177.1), Cassava virus C (CsVC, YP_003104770.1), Ourmia melon virus (OuMV, YP_002019757.1) and Epirus cherry virus (EpCV, YP_002019754.1). Evolutionary analysis was conducted using the software MEGA6 [30]. Multiple sequence alignments of amino acid sequences of viral RdRps were obtained using MUSCLE (Multiple sequence comparison by log-expectation, [7]) using default parameters. Phylogenetic relationships were inferred using the Maximum Likelihood method based on the $\mathrm{WAG}+\mathrm{G}+\mathrm{F}$ protein evolution model [35], and a bootstrap phylogeny test with 1,000 replicates. All positions with less than 50\% site coverage were eliminated. 


\section{Figure S1}

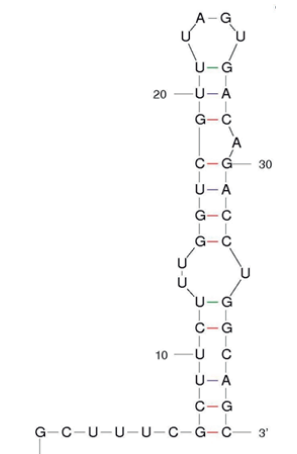

MOLV1 5'UTR ( $\Delta \mathrm{G}-10.00)$

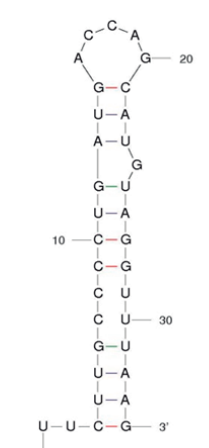

MOLV1 3'UTR ( $\Delta \mathrm{G}-6.30)$

Fig. S1. Predicted secondary structure of the 5' (left) and 3' (right) UTRs of MOLV1 with their corresponding $\Delta \mathrm{G}$ in $\mathrm{kcal} / \mathrm{mol}$. 